\title{
Long-term results after elective laparoscopic surgery for colorectal cancer in octogenarians
}
Benjamin WP Rossi ${ }^{1}$, Peter Labib ${ }^{1}$, Elizabeth Ewers ${ }^{1}$, Samantha Leong ${ }^{1}$, Mark Coleman ${ }^{1}$, Sebastian Smolarek ${ }^{1}$

\begin{abstract}
${ }^{1}$ Colorectal Department, University Hospital Plymouth NHS Trust
\end{abstract}
Corresponding author: Benjamin Rossi, Derriford Hospital, Plymouth, PL6 8DH, b.rossi@nhs.net 


\begin{abstract}
Background:

The aim of this study was to investigate the short and long term outcomes after elective laparoscopic surgery for colorectal cancer patients over 80 years of age.
\end{abstract}

\title{
Methods:
}

This was a retrospective study of all patients of 80 and above, who underwent elective colorectal resection, between January 2007 and January 2016. Data were analysed from a prospectively collected cancer database and cross checked with patient records. Determinants of survival were analysed using log rank test and Kaplan-Meier curves.

\section{Results:}

We identified 293 patients; 110 underwent laparoscopic surgery (LPS). LPS had significantly better overall survival $(p=0.0065)$, disease free survival $(p=0.006)$. The LPS group also had a shorter length of stay- 9 vs. 11 days ( $p<0.00001), 90$ day mortality - 5.5 vs. 13.7 per cent $(p=0.03)$ and required fewer blood transfusions 22.7 vs.40.4 per cent ( $p=0.002$ ), when compared to open surgery (OPS). There was no difference in 30 day mortality 1.8 vs. 4.9 per cent $(p=0.22)$, anastomotic leakage 2.3 vs 6 per cent $(p=0.20)$ or post-operative complication rates 44.5 vs. 50.8 per cent $(p=0.30)$.

\section{Conclusions:}

Laparoscopic surgery for patients in their $80 \mathrm{~s}$ is characterised by better overall and disease free survival compared to open procedures and is associated with shorter postoperative length of stay, and significantly lower 90 day mortality. Patients operated on laparoscopically also required fewer postoperative blood transfusions.

Keywords: Laparoscopic surgery, colorectal cancer, over 80s 


\section{Introduction}

Colorectal cancer (CRC) is the second leading cause of cancer related death in developed countries. Every year 412000 people are diagnosed with this condition, and 207000 patients die of the disease (1). One of the risk factors for CRC is advanced age (2). Taking into account this prolonged life in developed countries, the incidence of colorectal cancer will continue to rise. Advanced age is associated with increased co-morbidities, which contribute to higher post-operative morbidity and mortality.

Laparoscopic procedures are now widely used within colorectal surgery and many would argue that it is now the standard approach that should be offered to patients (3). Laparoscopic surgery is known to reduce respiratory complications (3); even in COPD patients, despite the perceived increased risks of pneumoperitoneum (4). Reduction in post-operative pain has also been confirmed (5); as has reduced length of stay in hospital. Long term benefits also include reduction in adhesions and consequent reduced need for re-operation (6). Mortality rates of elective colorectal surgery have been decreasing for many years (7), with an even greater reduction for laparoscopic resections (7) as low as $0.5 \%$ in some centres (8). However, in the older population this increases with a 30 - and 90 -day mortality as high as $4 \%$ and $10 \%$ respectively with only three-quarters of patients surviving to 1 year (9).

The benefits of laparoscopic surgery in patients over 80 years of age are still unclear; it may be that specific patients benefit, but as a general population it is thought that the increased duration of a laparoscopic operation (10) may be detrimental, thus making it less worthwhile. However, as laparoscopic surgery improves some studies dispute this fact (11), and it seems this variable is likely to be operator and centre dependent.

There is also currently no evidence that laparoscopic surgery is superior to open surgery in terms of oncological outcome. The most recent Cochrane Database systematic review showed similar fiveyear survival, overall survival and local recurrence rates (12). To date there is no published data for oncological outcomes after laparoscopic surgery for colorectal cancer in octogenarians. The aim of this retrospective review was to investigate the short and long term outcomes after elective laparoscopic surgery for colorectal cancer patients over 80 years of age.

\section{Materials and Methods}

All patients aged 80 and above, who underwent elective colorectal resection, between January 2007 and January 2016 for colorectal malignancy were included in this observational cohort study. Due to 
the retrospective nature of this study formal patient consent was not required, however, the project was approved by the Plymouth Hospital NHS Trust Audit department and the local Caldicott Guardian. All patients undergoing colorectal resection for cancer were identified from both the prospectively maintained colorectal oncology databases and hospital performance database, and verified from theatre records, hospital patient management systems and patient records. The study was reported in accordance with STrengthening the Reporting of Observational studies in Epidemiology (STROBE) methodology for observational studies (Figure 1) (13).

All patients were discussed formally at the hospital colorectal multidisciplinary team (MDT) meeting prior to treatment. Tumours were initially staged at presentation with chest, abdominal and pelvic computerised tomography (CT). After the MDT meeting all patients were assessed clinically in the MDT clinic with support from a specialist cancer nurse. At the time of that review, patients were carefully counselled about the potential risks and benefits of operative treatment as well as any alternative treatment options, including neo-adjuvant therapy. All patients with rectal cancer were additionally staged with pelvic MRI and rigid sigmoidoscopy for measurement of the distance of the tumour from the anal verge. In our unit both open and laparoscopic surgery are performed; the decision on which technique was used was made by the patient and consultant, based on patient preference and consultant experience with the technique. If there was no patient preference the patient was allocated to the next available theatre slot.

Pre-operative data including chemotherapy regimens, use of pre-operative radiotherapy and chemotherapy was recorded. All rectal cancer procedures complied with the principles of total mesorectal excision (TME) or partial mesorectal excision (PME). Anastomotic leakage was defined as clinical evidence of a defect of the integrity of the intestinal wall at the anastomotic site or presence of a pelvic abscess adjacent to the anastomosis.

Data regarding the primary origin of the tumour were collected alongside that regarding tumour type. Complete resection (RO) was defined pathologically as the absence of tumour cells within $1 \mathrm{~mm}$ or greater of the resection margin. Microscopic residual disease (R1) was defined as a presence of the tumour cells of less than $1 \mathrm{~mm}$ from the resection margin. Histopathology results were categorised according to whether the patient received neo-adjuvant therapy (ypT) or had proceeded straight to surgery (pT). We used TNM version 5 for staging. Surgical outcome measures were recorded and included: postoperative length of hospital stay; 30-day operative morbidity as well as 30 and 90 day mortality respectively. Surgical complications were classified using the Clavien-Dindo grading system (14). 
Where adjuvant chemotherapy was indicated a standard regimen for Stage III (Dukes C) colorectal cancer was used as per NICE guidelines, including capcetabine alone or oxaliplatin in combination with 5 fluorouracyl and folonic acid. Suggestion to start adjuvant chemotherapy was made at MDT meeting after reviewing the patients' post-operative histopathology results, followed by careful discussion between a specialist Gl oncologist and the patient. The final decision was made after the discussion based on patient preference.

For patients with rectal cancer a NICE recommendation regarding radiotherapy was used. Patients with T3b cancer on radiological staging, patients with suspicious lymph node without threatening the surgical margin and patients with the presence of extramural vascular invasion were considered as moderate risk and were considered for short course radiotherapy (SCRT) (5x5 Gy) with immediate surgery within 5 days. Patients with a threatened or breached resection margin or with a low tumour involving the inter-sphincteric plane or levator involvement were considered for long course radiotherapy (LCRT). The final decision was made after careful patient counselling by a specialist GI Oncologist, taking the patient's preference into account. Patients were followed up routinely at regular intervals for an indefinite length of time in a colorectal clinic. Patients were seen routinely every 6 months for two years and every 12 months afterwards up to year 5. A CT was performed at the end of any adjuvant treatment and also after year one and two. Decision about performing CT at year 5 was left to the discretion of the surgeon. After one year patients underwent a colonoscopy. If patients were not suitable for colonoscopy a CT colonography with thorax was performed instead. Decision regarding 5 year colonoscopy depended on the patients' general health.

The primary outcome was overall survival, which was defined as the time from the date of operation to the date of death or date of last follow up. Secondary endpoints were 5 year overall survival, disease free survival, operating time, conversion rate, blood loss, length of hospital stay, morbidity and mortality within 30 and 90 days after surgery, and histopathological outcomes (including completeness of the resection, circumferential, proximal, and distal resection margins, and number of resected lymph nodes). We also recorded anastomotic leakage and need for post-operative transfusion.

Statistical analyses were performed using the StatsDirect version 3.0 for Windows (StatsDirect Ltd. UK) and MedCalc (MedCalc Software bvba). Survival curves were calculated by the Kaplan-Meier method and differences between curves compared using the Wilcoxon log rank test. The results were presented as a Hazard Ratio and 95\% Confidence Interval. Other comparisons between treatments were done with the Mann-Whitney $\mathrm{U}$ test for continuous or ordered categorical data or the chi $^{2}$ test, if appropriate. $P=0.05$ (two-sided) was considered the limit of significance. 


\section{Results}

From January 2007 to January 2016, 293 patients above 80 years of age underwent operative treatment for colorectal cancer. 110 (37.5 per cent) patients were treated using laparoscopic approach (LPS) and 183 (62.5 per cent) open (OPS). 8.2 per cent (9/110) of patients in the LPS group were converted to an open procedure.

The median age was 88 (range 80-91) years. There were 144 male patients - 53 in LPS and 91 in the OPS group. The median Charlson Comorbidity Index (CACl) score was 16 (range 12-45). There was no difference in $\mathrm{CACl}$ between the groups ( $p=0.8)$. Patient demographics are displayed in Table 1.

The overall five year survival rates were 48.1 per cent - 57.9 per cent in the LPS and 45 per cent in the OPS group respectively (Table 2). There was a significant difference in overall survival rate (OS) between patients treated with LPS vs OPS ( $p=0.007$ ) [HR 0.5795 per cent $\mathrm{Cl} 0.4-0.8$ ] (Figure 2) and in disease free survival (DFS) ( $p=0.006$ ) [HR 0.5795 per cent $\mathrm{Cl} 0.4-0.8$ ] (Figure 3). 12.6 per cent $(23 / 193)$ of patients developed recurrence in the OPS group vs 8.3 per cent $(9 / 110)$ in the LPS group. There was also a difference in OS after exclusion of patients who died within 30 days from the operation ( $p=0.015)$ [HR 1.7195 per cent $\mathrm{Cl} 1.2$ - 2.5].

There were 151 right colon cancers - 54 in LPS and 97 in the OPS group, 56 Left colon cancers -25 in the LPS group and 31 in the OPS group and 86 rectal cancers - 31 in the LPS group and 55 in the OPS group. There was no difference in survival between left and right colon cancers $(p=0.75)$ and between rectal and colon cancers $(p=0.49)$. The exact numbers of operations are displayed in Table 3.

Complete resection (R0) was achieved in 263 ( 89.8 per cent) patients -90.9 per cent in the LPS group and 84.5 per cent in the OPS group, with no statistical difference between the groups $(p=0.7)$. The median number of lymph nodes harvested were 17 (range 4-43) for the LPS group and 18 (range 4-56) for the OPS group ( $p=0.7)$.

21 patients in the LPS group and 30 in the OPS group had stage I cancer, 55 and 85 respectively stage II and 34 and 67 stage III. Exact cancer staging for both groups is displayed in Table 4.

78.2 per cent $(86 / 110)$ of patients in the LPS group had an anastomosis vs 80.9 per cent $(148 / 193)$ in the OPS group ( $p=0.68$ ). $2 / 86$ ( 2.3 per cent) patients in the LPS group developed anastomotic leak vs $9 / 149$ (6 per cent) in the OPS group $(p=0.20)$. 
44.5 per cent $(49 / 110)$ of patients in the LPS group developed post-operative complications vs 50.8 per cent $(93 / 183)$ in the OPS group $(p=0.3)$. 30 day mortality was 1.8 per cent vs 4.9 per cent in the LPS group and OPS group respectively $(p=0.27)$ There was a difference in 90 day mortality -5.5 per cent for the LPS group vs. 13.7 per cent in the OPS group ( $p=0.03$ ). 25/110 patient in the LPS group and 74/183 in the OPS group required a post-operative blood transfusion. Patients in the LPS group required significantly less post-operative transfusion $(p=0.02)$. The median length of stay (LOS) in the LPS group was 9 days (range 2-85) and was statistically shorter compared with 11 days in the OPS group (range 3-81) $(p<0.00001)$ (Table 5).

There were 101 patients with stage III colorectal cancer of whom 12 received adjuvant chemotherapy (12 per cent). There was no difference in OS $(p=0.93)$ between the chemotherapy group and patients who did not receive it. Due to the small number of recurrences we were unable to compare DFS.

9 per cent of patients (8/86) received SCRT and 2 per cent (2/86) LCRT prior to rectal surgery. For statistical purposes we calculated these patients together. There was no difference in OS $(p=0.64)$ and DFS ( $p=0.85$ ) between patients with pre op radiotherapy and patients who were treated by surgery alone.

Logistic regression modelling was used to check for variation between the groups which showed there was no statistical significance between the overall demographic data. Analysis of the mortality risk factors showed that higher age, higher ASA score, more advanced cancer and higher number of transfusions were the negative risk factors influencing mortality, whereas LPS was a positive factor. (Table 6)

\section{Discussion}

The influence of laparoscopic surgery on the outcomes after colorectal procedures has previously been investigated by randomised control trials (15-17). These trials showed that laparoscopic surgery was associated with similar disease-free and overall survival rates as open surgery $(16,17)$ with favourable short-term outcomes, such as less pain, reduced blood loss, and improved recovery time (15). These studies, however, included younger groups of patients. Our study has similar findings in relation to length of stay and faster recovery, favouring the laparoscopic group. Interestingly our mean length of stay after laparoscopic surgery was 9 days, which is much shorter than previously reported in other studies for elderly populations (18). Hinoi et al (18) reported mean LOS after laparoscopic colorectal surgery as long as 12 days. This is even longer than the length of 
stay in our open surgery group. The reason for this might be in the wider acceptance of ERAS protocol into clinical practice in our unit.

In our study laparoscopic surgery was also associated with less significant blood loss and a decreased number of postoperative blood transfusions. This is one of the most important factors in octogenarians. Perioperative blood transfusions were associated with a worse overall outcome and higher mortality. Similar findings were also reported by different authors in younger patient populations (19-23); this association is even stronger in patients of 80 years and older. This may contribute to the findings that 90 day mortality was significantly lower in the laparoscopic group. Interestingly the 30 day mortality was also lower in the laparoscopic group, but without statistical significance. The reason for this is most likely the recent advances in perioperative and anaesthetic care. This contributes to a lower number of deaths in the early post-operative period, which pushes mortality outside the 30 day time frame. A large scale population based UK cohort study from 2013 (24) suggested that 90-day mortality is a preferred indicator of outcome and also correlated more strongly with later mortality results up to 1 year. For this reason 90 day mortality is now a part of a national colorectal cancer audit (25). Our study also showed that short term survival rates are particularly important in the over 80 s age group, where overall survival is lower than younger groups due the higher number of co-morbidities present.

One of the most important findings in our study is that laparoscopic surgery can influence oncological outcome in octogenarians. Patients operated on laparoscopically have better overall and disease free survival. The reason for this is unknown, but could be explained by the significantly higher impact on the body produced by open surgery. This can in turn disturb the gentle homeostasis between the host and the cancer, and decrease the immunological response. The immune response in elderly people is weaker than in the younger population due to poorer functional reserve and higher number of co-morbidities. If you add to this the stress related to an operation it can make patients more prone to recurrence and a poorer oncological outcome. A systematic review from 2004 looked at the stress response to laparoscopic surgery (26). It found that although an early response to $\mathrm{CO} 2$ insufflation was reduced peritoneal macrophage function, which reduces peritoneal immunity, the overall longer term systemic effects on immune response were of significant benefit compared to open surgery. This was mainly due to the increased stress response of open surgery because of the adverse effect on cytokine production and cell mediated immune response. Open surgery also increases serum levels of markers of inflammation including IL-6 and CRP. 
Oncological outcomes in octogenarians were previously investigated by Japan's Laparoscopic Group (18). In contrast to our study they were not able to show a difference in Overall Survival and Disease Free Survival between Laparoscopic and Open surgical groups. A recent meta-analysis of long term outcomes of laparoscopic surgery in elderly patients showed that laparoscopic surgery was associated with higher 3 year survival rates compared to open surgery with no difference in 5 year survival. However, this meta-analysis included patients of 65 years and above, with only 3 studies investigating octogenarians (27); hence the interpretation of the result is difficult.

Our study showed that overall complication rates were not significantly different between the groups, although the percentage was lower in the LPS group. This lack of significance did not affect the mortality rates in our cohort, although previous studies have suggested that major complications do affect long term survival (28). This may again be particular to the over 80 s age group, due to the higher co-morbidity status.

The limitation of this study is its retrospective nature, which may lead to selection bias, compared to the more rigourous methodology of a randomised trial. We were not able to retrieve some of the preoperative determinates which may influence morbidity and mortality such as BMI and smoking status as we would have with a prospective trial. The other limitation of this study is that there was no strict criteria for patient selection to open or laparoscopic surgery. This choice was left to the discretion of operating surgeon. In our institution there are still some surgeons who prefer open approach and do not perform laparoscopic surgery. The numbers of laparoscopic operations also steadily increased during the time of the study. Despite this both groups remain homogenous in terms of the patients' demographic data, as well as cancer stage and its distribution in the colon.

The obvious strengths of our study are the homogenous study group in terms of demographics, type of operation and cancer staging. Also the high numbers in each group leading to significant results in important areas.

\section{Conclusions}

Laparoscopic surgery for patients in their $80 \mathrm{~s}$ is characterised by better overall and disease free survival compared to open procedures and is associated with shorter postoperative length of stay, and significantly lower 90 day mortality. Patients operated laparoscopically also required fewer postoperative transfusions. We feel this study demonstrates that laparoscopic surgery could be considered as a primary approach to all patients over 80 years of age unless otherwise contraindicated. 


\section{Disclosures}

Benjamin Rossi - Nothing to disclose

Peter Labib - Nothing to disclose

Elizabeth Ewers - Nothing to disclose

Samantha Leong - Nothing to disclose

Mark Coleman - Nothing to disclose

Sebastian Smolarek - Nothing to disclose

\section{References}

1. Zavoral M, Suchanek S, Zavada F, Dusek L, Muzik J, Seifert B, Fric P (2009). Colorectal cancer screening in Europe. World J Gastroenterol. 15(47):5907-15.

2. Spann SJ, Rozen P, Young GP, Levin B (2002). Colorectal cancer: how big is the problem, why prevent it, and how might it present? In: Rozen P, Young GP, Levin B, Spann SJ. (eds). Colorectal Cancer in Clinical Practice. London: Martin Dunitz Ltd; p. 1-18.

3. Keller DS, Delaney CP, Hashemi L, Haas EM (2016). A national evaluation of clinical and economic outcomes in open versus laparoscopic colorectal surgery. Surg Endosc. 30(10):4220-8.

4. Sujatha-Bhaskar S, Alizadeh RF, Inaba CS, Koh CY, Jafari MD, Mills SD, Carmichael JC, Stamos MJ, Pigazzi A (2018). Respiratory complications after colonic procedures in chronic obstructive pulmonary disease: does laparoscopy offer a benefit? Surg Endosc . 32(3):12801285.

5. Schwenk W, Haase O, Neudecker J, Müller JM (2005). Short term benefits for laparoscopic colorectal resection. Cochrane Database Syst Rev. 20;(3):CD003145.

6. Ha GW, Lee MR, Kim JH (2016). Adhesive small bowel obstruction after laparoscopic and open colorectal surgery: a systematic review and meta-analysis Am J Surg.212(3):527-36.

7. Lee MG, Chiu CC, Wang CC, Chang CN, Lee SH, Lee M, Hsu TC, Lee CC (2017). Trends and Outcomes of Surgical Treatment for Colorectal Cancer between 2004 and 2012 - an Analysis using National Inpatient Database. Sci Rep. 17;7(1):2006. 
8. Juo YY, Hyder O, Haider AH, Camp M, Lidor A, Ahuja N (2014). Is minimally invasive colon resection better than traditional approaches?: First comprehensive national examination with propensity score matching. JAMA Surg.149(2):177-84.

9. Mäkelä JT, Klintrup KH, Rautio TT (2017). Mortality and Survival after Surgical Treatment of Colorectal Cancer in Patients Aged over 80 Years. Gastrointest Tumors. 4(1-2):36-44.

10. Trastulli S, Cirocchi R, Listorti C, Cavaliere D, Avenia N, Gullà N, Giustozzi G, Sciannameo F, Noya G, Boselli C (2012). Laparoscopic vs open resection for rectal cancer: a meta-analysis of randomized clinical trials. Colorectal Dis.14(6):e277-96.

11. Pinto RA, Ruiz D, Edden Y, Weiss EG, Nogueras JJ, Wexner SD (2011). How reliable is laparoscopic colorectal surgery compared with laparotomy for octogenarians? Surg Endosc.25:2692-2698.

12. Vennix S, Pelzers L, Bouvy N, Beets GL, Pierie JP, Wiggers T, Breukink S (2014). Laparoscopic versus open total mesorectal excision for rectal cancer. Cochrane Database Syst Rev.(4):CD005200.

13. Fernandez E, STROBE group (2005). [Observational studies in epidemiology (STROBE).] Med Clin (Barc).125:43-48.

14. Dindo, D., Demartines, N., Clavien, P.A (2004). Classification of surgical complications: a new proposal with evaluation in a cohort of 6336 patients and results of a survey. Ann Surg. 240:205-213.

15. Veldkamp R, Kuhry E, Hop WC, Jeekel J, Kazemier G, Bonjer HJ, Haglind E, Påhlman L, Cuesta MA, Msika S, Morino M, Lacy AM; COlon cancer Laparoscopic or Open Resection Study Group (COLOR) (2005). Laparoscopic surgery versus open surgery for colon cancer: shortterm outcomes of a randomised trial. Lancet Oncol. 6:477-484.

16. Lacy AM, Garcia-Valdecasas JC, Delgado S, Castells A, Taurá P, Piqué JM, Visa J (2002). Laparoscopy-assisted colectomy versus open colectomy for treatment of non-metastatic colon cancer: a randomised trial. Lancet.359:2224-2229.

17. Buunen M, Veldkamp R, Hop WC, Kuhry E, Jeekel J, Haglind E, Påhlman L, Cuesta MA, Msika S, Morino M, Lacy A, Bonjer HJ (2009). Survival after laparoscopic surgery versus open surgery for colon cancer: long-term outcome of a randomised clinical trial. Lancet Oncol.10(1):44-52.

18. Hinoi $T$, Kawaguchi $Y$, Hattori $M$, Okajima $M$, Ohdan $H$, Yamamoto $S$, Hasegawa $H$, Horie $\mathrm{H}$, Murata K, Yamaguchi S, Sugihara K, Watanabe M 
(2015). Laparoscopic versus open surgery for colorectal cancer in elderly patients: a multicenter matched case-control study. Ann Surg Oncol. 22(6):2040-50.

19. Amato AC, Pescatori M (1998). Effect of perioperative blood transfusions on recurrence of colorectal cancer: meta-analysis stratified on risk factors. Dis Colon Rectum.41(5):570-585.

20. Burrows L, Tartter P (1982). Effect of blood transfusions on colonic malignancy recurrence rate. Lancet.2(8299):662.

21. Blumberg N, Agarwal MM, Chuang C (1985). Relation between recurrence of cancer of the colon and blood transfusion. Br Med J (Clin Res Ed).290(6474):1037-1039.

22. Edna TH, Bjerkeset $T$ (1998). Perioperative blood transfusions reduce long-term survival following surgery for colorectal cancer. Dis Colon Rectum.41(4):451-459.

23. Tartter PI (1988). Blood transfusion and infectious complications following colorectal cancer surgery. Br J Surg.75(8):789-792.

24. Byrne BE, Mamidanna R, Vincent CA and Faiz O (2013). Population-based cohort study comparing 30- and 90-day institutional mortality rates after colorectal surgery. Br J Surg. 100(13):1810-7.

25. National Bowel Cancer Audit. Performance Indicators Description. Available from: www.nboca.org.uk [Accessed $9^{\text {th }}$ June 2018].

26. Buunen M, Gholghesaei M, Veldkamp R, Meijer DW, Bonjer HJ, Bouvy ND (2004). Stress response to laparoscopic surgery: a review. Surg Endosc. 18(7):1022-8.

27. Wang F, Yu Z, Zhao M, Maomin S (2017). Long term outcomes of laparoscopic vs. open surgery for colorectal cancer in eldery patients: A meta-analysis. Molecular and Clinical Oncology.7:771-776.

28. Odermatt M, Miskovic D, Flashman K, Khan J, Senapati A, O'Leary D, Thompson M, Parvaiz A (2015). Major postoperative complications following elective resection for colorectal cancer decrease long-term survival but not the time to recurrence. Colorectal Dis. 17(2):141-9.

\section{Figures}

\section{Flow chart of study design}

\section{Kaplan-Meier curve of overall survival (OS)}

\section{Kaplan-Meier curve of disease free survival (DFS)}

